\title{
Evaluation of prostate-specific antigen density in the diagnosis of prostate cancer combined with magnetic resonance imaging before biopsy in men aged 70 years and older with elevated PSA
}

\author{
YOSHINORI YANAI $^{1}$, TAKEO KOSAKA ${ }^{1}$, HIROSHI HONGO ${ }^{1}$, KAZUHIRO MATSUMOTO ${ }^{1}$, \\ TOSHIAKI SHINOJIMA ${ }^{1}$, EIJI KIKUCHI ${ }^{1}$, AKIRA MIYAJIMA ${ }^{1}$, RYUICHI MIZUNO ${ }^{1}$, \\ SHUJI MIKAMI ${ }^{2}$, MASAHIRO JINZAKI ${ }^{3}$ and MOTOTSUGU OYA ${ }^{1}$ \\ ${ }^{1}$ Department of Urology, ${ }^{2}$ Division of Diagnostic Pathology and ${ }^{3}$ Division of Diagnostic Radiology, \\ Keio University School of Medicine, Tokyo 160-8582, Japan
}

Received March 29, 2018; Accepted September 3, 2018

DOI: $10.3892 / \mathrm{mco} .2018 .1725$

\begin{abstract}
There is an increasing proportion of individuals aged 70 years and older, as well as an increasing life expectancy worldwide. The present study may guide the management of older patients with elevated prostate specific antigen (PSA). The medical records of 241 older men aged $>70$ years who underwent multiparametric magnetic resonance imaging (mpMRI) before prostate biopsy (PBx) at our institution were reviewed retrospectively. Multiple variables were evaluated as predictors for the diagnosis of prostate cancer $(\mathrm{PCa})$. The variables included serum PSA level, digital rectal examination, size of region of interest on mpMRI, prostate volume and PSA density. PCa was positive in $162(67.2 \%)$. Prostate volume and PSA density were significant $\mathrm{PCa}$ predictors $(\mathrm{P}<0.001)$. In patients aged $70-75$ and $>75$ years, PSA density was significantly higher in patients with $\mathrm{PCa}(0.21 \mathrm{ng} / \mathrm{ml} / \mathrm{cc}, \mathrm{P}=0.014$ and $0.24 \mathrm{ng} / \mathrm{ml} / \mathrm{cc}, \mathrm{P}<0.001$, respectively). Similarly, PSA density was significant higher in patients with significant $\mathrm{PCa}(0.24 \mathrm{ng} / \mathrm{ml} / \mathrm{cc}, \mathrm{P}=0.004$ and $0.29 \mathrm{ng} / \mathrm{ml} / \mathrm{cc}, \mathrm{P}<0.001$, respectively). The cut-off value of PSA density was calculated using receiver operating characteristic curves. Area under curve of PSA density was 0.698 , and the best cut-off value was $0.20 \mathrm{ng} / \mathrm{ml} / \mathrm{cc}$. These results indicate that the combination of PSA density with mpMRI before PBx is a helpful method and can be a decision-making model for a selection of $\mathrm{PBx}$.
\end{abstract}

Correspondence to: Dr Takeo Kosaka, Department of Urology, Keio University School of Medicine, 35 Shinanomachi, Shinjuku, Tokyo 160-8582, Japan

E-mail: takemduro@gmail.com

Key words: elderly men, magnetic resonance imaging, prostate cancer screening, prostate-specific antigen density, prostate biopsy

\section{Introduction}

Older men usually have a shorter life expectancy, a higher risk of competing causes of mortality, and a greater risk of potential harm from screening for prostate cancer (PCa) (1). It is evident that there is an increasing proportion of individuals aged 70 years and older, as well as an increasing life expectancy worldwide (2). The life expectancy of men aged 70 years old in America is estimated to be 14 years compared with 15 years in Japan (3). The American Urological Association does not recommend routine prostate-specific antigen (PSA) screening in men $>70$ years old or any man with less than a 10-15 year life expectancy (4). In Japan, however, there is no clear consensus among urologists on how to manage older men aged 70 years and older with elevated PSA.

The classical risk factors for PCa include elevating PSA and positive digital rectal examination (DRE). The current standard of care practice for a diagnosis of $\mathrm{PCa}$ is a needle biopsy of the prostate (PBx) (5). However, a number of criticisms have been directed against PSA-based screening for $\mathrm{PCa}$, which lead to overdiagnosis and overtreatment in certain cases (6). Recent screening guidelines do not recommend PSA screening alone, especially in older men (7). The risks of PBx include pain, urinary retention, hematuria, hemospermia, and, most significantly, urinary tract infection (UTI). The reported incidence for UTIs is $2-6 \%$, and for severe sepsis is $0.2-2 \%$ (8). Therefore, other approaches may be necessary to prevent unnecessary biopsies, especially for older men.

Prediction methods were developed to reduce unnecessary biopsies, to avoid false-negative biopsies while still detecting most clinically important PCa cases before biopsy (9). These are more accurate than PSA screening alone. An ideal predictive tool would be a reliable imaging method that excludes inter-observer variability such as in DRE and transrectal ultrasound (TRUS) prostate images. Multiparametric magnetic resonance imaging (mpMRI) is now widely used in diagnosis, staging after diagnosis, and treatment planning of PCa (10-12). We reported previously that MRI positivity can independently predict biochemical recurrence after radical prostatectomy (13). Recently, the European Society of Urogenital Radiology 


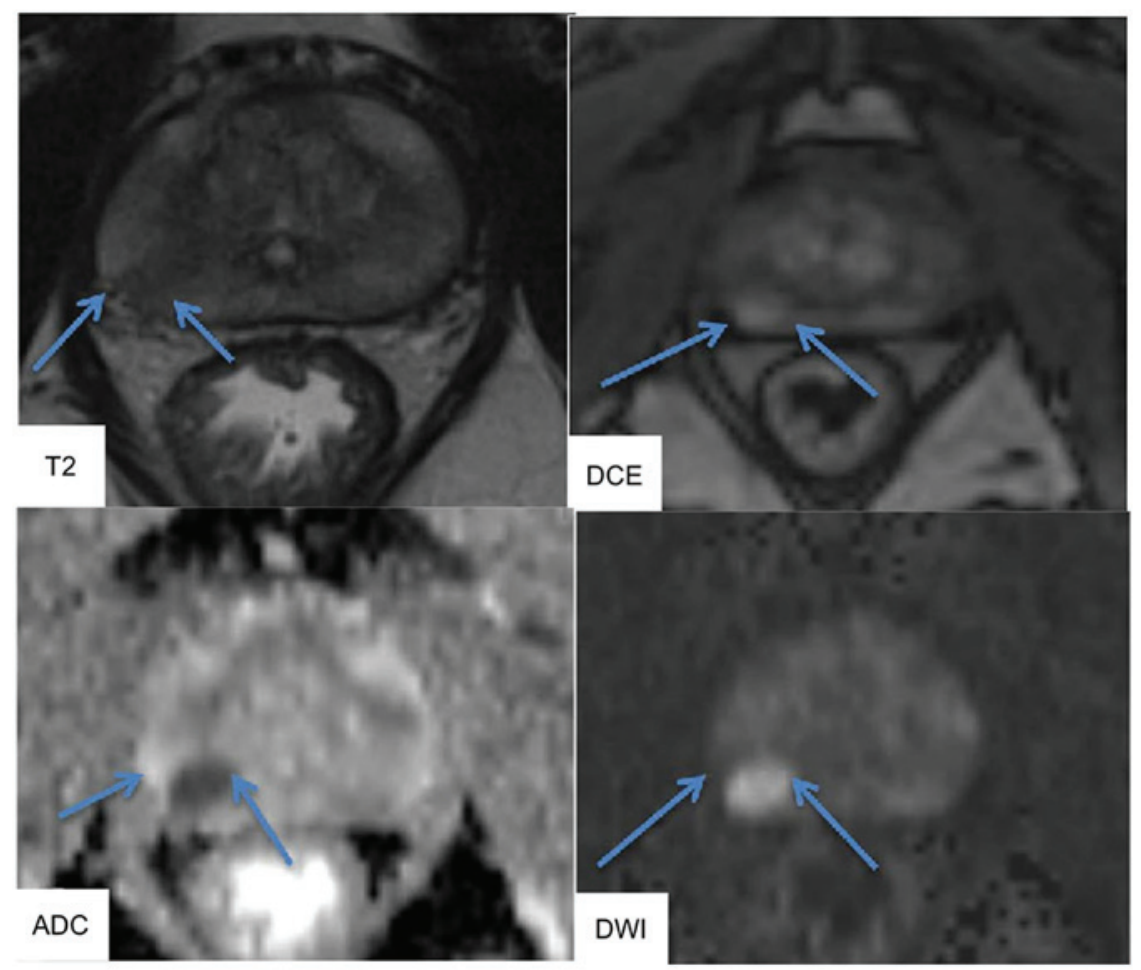

Figure 1. Typical images of multiparametric magnetic resonance imaging. T2-weighted imaging (T2WI) and diffusion-weighted imaging (DWI) were performed using an endorectal coil at a 3.0 tesla scanner. T2WI showed a hypointense area in the right peripheral zone (arrow). DWI and dynamic contrast-enhanced (DCE) were reported to provide higher diagnostic sensitivity. DCE and DWI showed area of rounded hyperintensity (arrow). DWI allowed apparent diffusion coefficient (ADC) maps to be calculated. The ADC map depicted a cancer focus in the right peripheral zone as a hypointense are (arrow).

(ESUR) proposed the Prostate Imaging Reporting and Data System (PI-RADS) to detect PCa (14). In addition to imaging, we reported that PSA density is also useful for distinguishing benign prostatic hypertrophy and PCa $(13,15)$.

The aim of the present study was to evaluate the role of PSA density after mpMRI before PBx, especially for older men, to avoid overscreening, overdiagnosis, and overtreatment.

\section{Patients and methods}

Study design. The medical records of 241 patients aged $>70$ years who underwent mpMRI before $\mathrm{PBx}$ at our institution between January 2012 and December 2016 were reviewed retrospectively in this study. Exclusion criteria was serum PSA level >20 ng/ml. Multiple variables were evaluated as predictors for the diagnosis of PCa. The variables and indications for biopsy included serum PSA level, DRE, size of region of interest (ROI) on mpMRI, prostate volume, and PSA density.

Imaging. At our institution, the mpMRI system is a 3.0-Tesla scanner (Signa Excite XI 1.5 and 3.0-Tesla, 32-channel torso-array coil). T2-weighted fast spin-echo imaging (T2WI, 3000/120, 4 NEX, $4 \mathrm{~min} 36 \mathrm{sec}, 3.4 \mathrm{~mm}$ slice, $0 \mathrm{~mm}$ space), diffused-weighted imaging (DWI, single-shot diffusion echo plannar imaging, $b=0,2,000 \mathrm{sec} / \mathrm{min}^{2}$ for whole pelvic, 0 , $1,500 \mathrm{sec} / \mathrm{min}^{2}$ for prostate, $4000 / 69.5$ (pelvis), 4000/58.3 (prostate), $1 \mathrm{~min} 24 \mathrm{sec}, 3 \mathrm{~min} 24 \mathrm{sec}$ ), and Gadolinium-dynamic contrast enhanced MRI (DCE, liver imaging with Volume Acceleration-flexible (LAVA-Flex), 4/1.1/12 ${ }^{\circ}, 15 \mathrm{sec}, 40,60$, and $180 \mathrm{sec}$ after i.v.) were performed. MRI could identify
Table I. Characteristics of all patients who underwent MRI before needle biopsy of the prostate.

\begin{tabular}{lc}
\hline Characteristic & $\begin{array}{c}\text { Overall }(\mathrm{N}=241) \\
\text { median } \pm \text { SD (95\% CI) }\end{array}$ \\
\hline Age, years & $76 \pm 4.3(70-85)$ \\
PSA, ng/ml & $7.2 \pm 3.6(4.0-15.5)$ \\
MRI ROI size, mm & $10.0 \pm 7.1(0.0-24.3)$ \\
Prostate volume, cc & $35.1 \pm 18.3(18.5-80.5)$ \\
PSA density, ng/ml/cc & $0.20 \pm 0.14(0.08-0.51)$ \\
\hline
\end{tabular}

ROI, region of Interest; MRI, magnetic resonance imagine; CI, confidence interval; SD, standard deviation; PSA, prostate specific antigen.

ROIs, and MR images were interpreted by experienced radiologists in accordance with ESUR PI-RADS v2.0. The typical images of mpMRI were shown in Fig. 1. T2WI was used for prostate cancer detection, localization and imaging. T2WI alone was sensitive but not specific for PCa and should be improved using DCE and DWI MRI. DWI allowed apparent diffusion coefficient (ADC) maps to be calculated, enabling qualitative and quantitative assessment of PCa aggressiveness.

Biopsy. All patients underwent TRUS-guided systematic biopsies of 12 cores that included lateral and mid lobar cores at the base, middle, and apex of each prostate lobes. All biopsy specimens were interpreted histopathologically by 
experienced pathologists according to the recommendations of the International Society of Urological Pathology (ISUP). Clinical significant PCa was defined as Gleason score $\geq 4+3=7$. We compared the MRI findings with the pathological findings retrospectively. At least one positive area on biopsy matched with each MRI lesion suspicious for cancer and vice versa were defined as matched. The absence of areas with pathological reports matching each MRI lesion was defined as unmatched.

Data analysis. Prostate volume in this study was measured in axial and sagittal images (height $\mathrm{x}$ width $\mathrm{x}$ depth/2) using both TRUS and MRI. PSA density was calculated by dividing serum PSA levels by prostate volume. Prostate volumes in TRUS were calculated by each urologist. All prostate volumes in MRI were calculated by one author. The factors evaluated for PBx included age, serum PSA level, index tumor size of ROI on MRI, prostate volume, and PSA density.

Statistical analysis. Statistical analyses were performed using the R Statistical Language version 3.0.2 and the SPSS version 23.0 statistical software packages. Chi-squared test was used to test the distribution of categorical variables, and the Mann-Whitney U test was used for continuous variables. We calculated the best-fit receiver operating characteristic (ROC) curve and the corresponding area under the ROC curve (AUC) estimates and $95 \%$ confidence interval (CI), then calculated the cut-off value of the PSA density. Spearman's rank correlation and linear regression were used to determine the relationship between TRUS based PSA density and MRI based PSA density. All reported P-values were two-sided with significance considered at $\mathrm{P}<0.05$.

\section{Results}

Patient characteristics. The median age of patients was 76 (range, 70-85) years, median serum PSA level was $7.2(95 \%$ confidence interval (CI), 4.0-15.5) ng/ml, median MRI ROI size was $10.0(95 \%$ CI, 0.0-24.3) $\mathrm{mm}$, median prostate volume was 35.1 (95\% CI, 18.5-80.5) cc, and PSA density was $0.20(95 \%$ CI, 0.08-0.51) ng/ml/cc (Table I). The correlation between TRUS-PSA density and MRI-PSA density was very strong $(r=0.873)$ and significant $(\mathrm{P}<0.001)$. There was no inter-observer error in the measurement of PSA density using TRUS and MRI.

Prostate cancer detection on mpMRI. In total, 221 patients had suspicious lesions for cancer in mpMRI (Table II). The median PSA density of these patients was $0.21 \mathrm{ng} / \mathrm{ml} / \mathrm{cc}$. Conversely, 20 patients did not have suspicious lesions for cancer in mpMRI, and their median PSA density was $0.16 \mathrm{ng} / \mathrm{ml} / \mathrm{cc}$, which was significantly lower $(\mathrm{P}=0.023)$.

Prostate cancer detection on biopsy. PCa was detected on biopsy in 163 patients (Table III). The median PSA density of these patients was $0.23 \mathrm{ng} / \mathrm{ml} / \mathrm{cc}$. Conversely, 78 patients showed no evidence of malignancy on biopsy, and their median PSA density was $0.16 \mathrm{ng} / \mathrm{ml} / \mathrm{cc}$, which was significantly lower $(\mathrm{P}<0.001)$.

Usefulness of PSA density. The ROC curve analysis for predicting PCa using mpMRI showed that the maximum AUC values of PSA, prostate volume, and PSA density were 0.507, 0.280 , and 0.698 , respectively (Fig. 2). These results indicated that PSA density could be a useful parameter to predict PCa. The PSA density of the maximum AUC value was $0.20 \mathrm{ng} / \mathrm{ml} / \mathrm{cc}$. The sensitivity, specificity, positive predictive value, and negative predictive value of PSA density threshold of $0.20 \mathrm{ng} / \mathrm{ml} / \mathrm{cc}$ were $63,71,82$, and $47 \%$, respectively.

Subgroup analyses. Patients with or without significant PCa did not differ significantly in their serum PSA level $(\mathrm{P}=0.089)$. Prostate volume of patients with significant $\mathrm{PCa}$ was significantly smaller $(\mathrm{P}<0.001)$. Calculated PSA density was significantly higher in patients with significant PCa with a median of $0.27 \mathrm{ng} / \mathrm{ml} / \mathrm{cc}$ vs. $0.18 \mathrm{ng} / \mathrm{ml} / \mathrm{cc}(\mathrm{P}<0.001)$. The median PSA density of patients with and without significant $\mathrm{PCa}$ at the age of 70 to 75 years differed significantly, at 0.24 and $0.17 \mathrm{ng} / \mathrm{ml} / \mathrm{cc}$, respectively $(\mathrm{P}=0.004)$. The median PSA density of patients with and without significant PCa over the age of 75 years also differed significantly, at 0.29 and $0.20 \mathrm{ng} / \mathrm{ml} / \mathrm{cc}(\mathrm{P}<0.001$, Fig. 3).

\section{Discussion}

Although mpMRI is increasingly used for PCa diagnosis, there is no consensus whether or not older men need a biopsy and how follow-up should be managed. In the present study, mpMRI combined with PSA density before biopsy for older men was useful for the selection of prostate biopsy. Until recently, previous reports have described the use of MRI before biopsy combined with PSA density $(16,17)$; however, no reports have focused on age. Kosaka et al reported that PSA density was a very effective predictor of clinically significant $\mathrm{PCa}$ in men aged 50 years and younger (18). Our results indicated that PSA density could also help predict the presence of $\mathrm{PCa}$ and significant PCa in men aged 70 years and older.

Compared with younger patients, older patients were more likely to present with advanced diseases and had a greater risk of death from PCa. However, older men have a shorter life expectancy and a higher risk of competing causes of mortality (19). In our study, 20 patients did not have suspicious lesions for cancer in mpMRI but underwent PBx. No significant PCa was detected in these 20 patients after PBx. Men without suspicious lesions for cancer in mpMRI could have avoided a biopsy. Men with lower PSA density have a lower risk of significant cancer, and these men could have avoided a biopsy. The cut-off value of PSA density was previously reported as 0.15 or $0.20 \mathrm{ng} / \mathrm{ml} / \mathrm{cc}(16,20,21)$, which was the PSA density of the maximum AUC values in our study.

Our retrospective study has several limitations. First, all elderly patients in the present study underwent PBx after MRI. Patients without lesions suspicious for PCa on mpMRI had already been excluded. Older men with lesions suspicious for $\mathrm{PCa}$ on mpMRI, who were too sick to undergo PBx, were also excluded. Second, with marked advances in mpMRI, further prospective studies considering prognosis could be more precise in defining the role of mpMRI combined with PSA density.

Despite these limitation, there are advantages in the present study. First, we focused on older men aged 70 years and older. Older men have a shorter life expectancy and prognosis can 
Table II. Clinical characteristics and predictors analysis of patients among MRI lesions suspicious and not suspicious for prostate cancer.

\begin{tabular}{|c|c|c|c|}
\hline & Suspicious for $\mathrm{PCa}$ & Not suspicious for $\mathrm{PCa}$ & \\
\hline Characteristic & $\mathrm{N}=221$ median $\pm \mathrm{SD}(95 \% \mathrm{CI})$ & $\mathrm{N}=20$ median $\pm \mathrm{SD}(95 \% \mathrm{CI})$ & P-value \\
\hline PSA, ng/ml & $7.4 \pm 3.7(3.9-16.3)$ & $6.7 \pm 2.2(4.6-12.0)$ & 0.216 \\
\hline MRI ROI size, mm & $12.0 \pm 6.5(5.0-25.0)$ & NA & NA \\
\hline Prostate volume, cc & $34.8 \pm 18.7(17.2-81.8)$ & $45.8 \pm 14.5(22.0-73.5)$ & 0.098 \\
\hline PSA density, ng/ml/cc & $0.21 \pm 0.14(0.08-0.51)$ & $0.16 \pm 0.08(0.09-0.42)$ & 0.023 \\
\hline
\end{tabular}

ROI, region of interest; MRI, magnetic resonance imagine; CI, confidence interval; SD, standard deviation; PSA, prostate specific antigen.

Table III. Patients' pathological characteristics.

\begin{tabular}{|c|c|c|c|}
\hline & PCa negative & PCa positive & \\
\hline Characteristic & $\mathrm{N}=78$ median $\pm \mathrm{SD}(95 \% \mathrm{CI})$ & $\mathrm{N}=163$ median $\pm \mathrm{SD}(95 \% \mathrm{CI})$ & P-value \\
\hline PSA, ng/ml & $7.5 \pm 3.7(3.6-15.3)$ & $7.2 \pm 3.6(4.1-16.4)$ & 0.858 \\
\hline MRI ROI size, $\mathrm{mm}$ & $10.0 \pm 5.6(0.0-20.0)$ & $10.9 \pm 7.5(0.0-28.0)$ & 0.057 \\
\hline Prostate volume, cc & $46.0 \pm 19.6(21.9-83.4)$ & $32.9 \pm 16.0(15.3-71.9)$ & $<0.001$ \\
\hline PSA density, $\mathrm{ng} / \mathrm{ml} / \mathrm{cc}$ & $0.16 \pm 0.11(0.07-0.43)$ & $0.23 \pm 0.15(0.10-0.51)$ & $<0.001$ \\
\hline
\end{tabular}

ROI, region of interest; MRI, magnetic resonance imagine; CI, confidence interval; SD, standard deviation; PSA, prostate specific antigen.

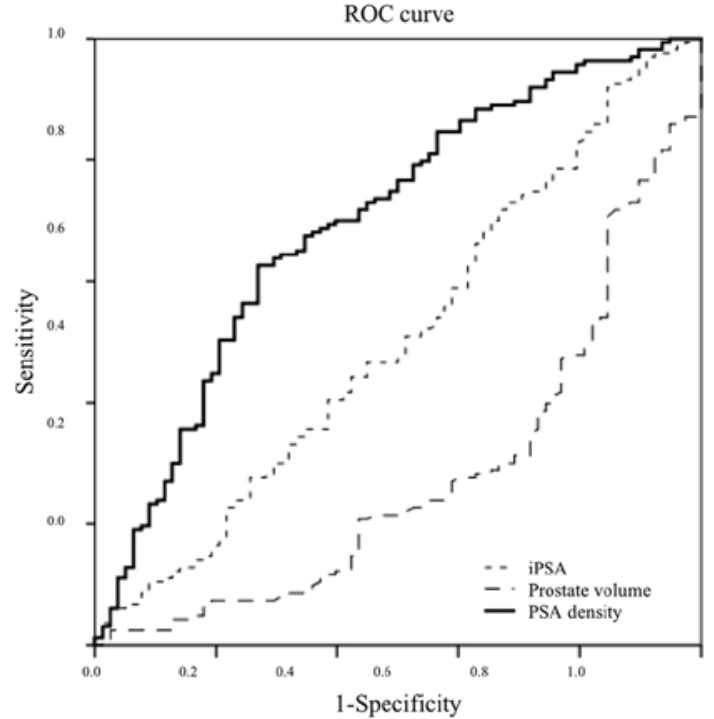

Figure 2. ROC curve analysis. The ROC curve analysis for predicting PCa using mpMRI showed that the maximum AUC values of PSA, prostate volume, and PSA density were $0.507,0.280$, and 0.698 , respectively. The PSA density of the maximum AUC values was $0.20 \mathrm{ng} / \mathrm{ml} / \mathrm{cc}$.

be evaluated more easily than in younger men. An additional study considering patient prognosis will be carried out in the future. There is a possibility that cancer in smaller prostates was more easily detected, and larger glands could be associated with difficulty in detecting cancer. Discussion of data on the correlation between prostate size and pathological features

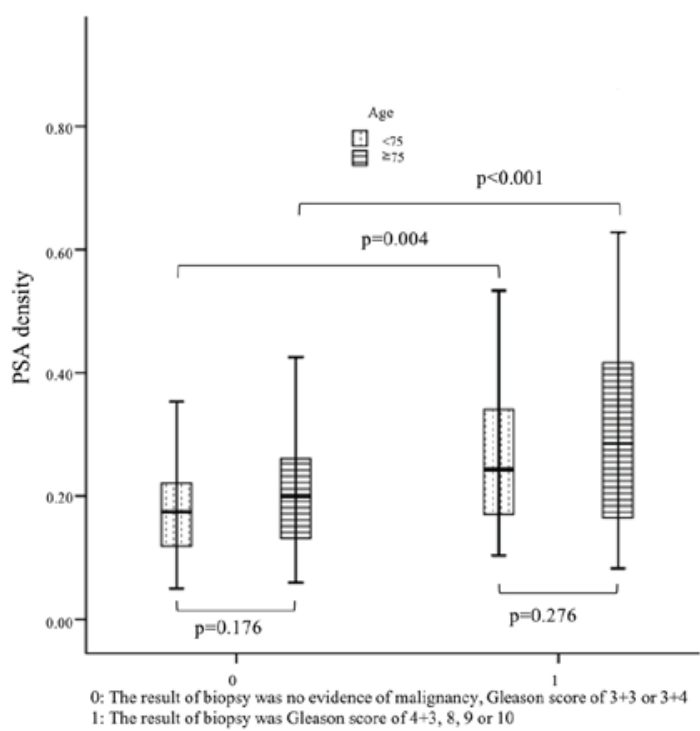

Figure 3. PSA density of patients with or without significant PCa aged $70-75$ years and $>75$ years. The median PSA density in patients aged 70-75 years with and without significant PCa differed significantly, at 0.24 and $0.17 \mathrm{ng} / \mathrm{ml} / \mathrm{cc}$, respectively $(\mathrm{P}=0.004)$. The median PSA density of patients aged $>75$ years with and without significant $\mathrm{PCa}$ also differed significantly, at 0.29 and $0.20 \mathrm{ng} / \mathrm{ml} / \mathrm{cc}(\mathrm{P}<0.001)$.

of PCa will be examined in an upcoming study. Second, it has been controversial whether to perform MRI before biopsy for men of all ages because of cost-effectiveness. For older men with a shorter life expectancy, however, the cost savings from avoiding unnecessary biopsies will offset the cost of MRI. 
In conclusion, PSA density combined with mpMRI before PBx performed significantly better compared with mpMRI or PSA density alone. The combination is a helpful method and can be a decision-making model for the selection of $\mathrm{PBx}$.

\section{Acknowledgements}

Not applicable.

\section{Funding}

No funding was received.

\section{Availability of data and materials}

The analyzed data sets generated during the study are available from the corresponding author on reasonable request.

\section{Authors' contributions}

YY and TK collected the data and wrote the manuscript. KM, RM and SM reviewed the slides and confirmed the pathological diagnoses. TS and MJ reviewed the images and confirmed the radiological diagnoses. YY and $\mathrm{HH}$ analyzed and interpreted the patient data regarding the clinical outcomes. AM, EK and MO provided the study concept and design, and revised the manuscript. The final version of the manuscript was read and approved by all authors.

\section{Ethics approval and consent to participate}

The postoperative prognostic and clinicopathological data were analyzed retrospectively in accordance with a protocol approved by the Keio University Institutional Review Board.

\section{Patient consent for publication}

Not applicable.

\section{Competing interests}

The authors declare that they have no competing interests.

\section{References}

1. Daskivich TJ, Fan KH, Koyama T, Albertsen PC, Goodman M, Hamilton AS, Hoffman RM, Stanford JL, Stroup AM and Litwin MS: Original research effect of age, tumor risk and comorbidity on competing risks. Ann Intern Med 158: 709-717, 2013.

2. Siegel RL, Miller KD and Jemal A: Cancer statistics, 2015. CA Cancer J Clin 65: 5-29, 2015.

3. Honorable T and Lew JJ: Social security. Available via DIALOG. https://www.ssa.gov/OACT/population/longevity.html. Accessed March 1, 2018

4. Smith RA, Manassaram-Baptiste D, Brooks D, Doroshenk M, Fedewa S, Saslow D, Brawley OW and Wender R: Cancer screening in the United States, 2015: A review of current American cancer society guidelines and current issues in cancer screening. CA Cancer J Clin 65: 30-54, 2015

5. Heidenreich A, Bastian PJ, Bellmunt J, Bolla M, Joniau S, van der Kwast T, Mason M, Matveev V, Wiegel T, Zattoni F, et al: EAU guidelines on prostate cancer. part 1: Screening, diagnosis, and local treatment with curative intent-update 2013. Eur Urol 65: 124-137, 2013
6. Lin K, Lipsitz R, Miller T and Janakiraman S; U.S. Preventive Services Task Force: Benefits and harms of prostate-specific antigen screening for prostate cancer: An evidence update for the U.S Preventive Services Task Force. Ann Intern Med 149: 192-199, 2008.

7. Carter HB, Albertsen PC, Barry MJ, Etzioni R, Freedland SJ, Greene KL, Holmberg L, Kantoff P, Konety BR, Murad MH, et al: Early detection of prostate cancer: AUA guideline. J Urol 190: 419-426, 2013.

8. Williamson DA, Barrett LK, Rogers BA, Freeman JT, Hadway P and Paterson DL: Infectious complications following transrectal ultrasound-guided prostate biopsy: New challenges in the era of multidrug-resistant Escherichia coli. Clin Infect Dis 57: 267-274, 2013.

9. Schröder F and Kattan MW: The comparability of models for predicting the risk of a positive prostate biopsy with prostate-specific antigen alone: A systematic review. Eur Urol 54: 274-290, 2008

10. Sonn GA, Chang E, Natarajan S, Margolis DJ, Macairan M, Lieu P, Huang J, Dorey FJ, Reiter RE and Marks LS: Value of targeted prostate biopsy using magnetic resonance-ultrasound fusion in men with prior negative biopsy and elevated prostate-specific antigen. Eur Urol 65: 809-815, 2014.

11. Pokorny MR, de Rooij M, Duncan E, Schröder FH, Parkinson R, Barentsz JO and Thompson LC: Prospective study of diagnostic accuracy comparing prostate cancer detection by transrectal ultrasound-guided biopsy versus magnetic resonance (MR) imaging with subsequent MR-guided biopsy in men without previous prostate biopsies. Eur Urol 66: 22-29, 2014.

12. Hoeks CM, Schouten MG, Bomers JG, Hoogendoorn SP, Hulsbergen-van de Kaa CA, Hambrock T, Vergunst $H$, Sedelaar JP, Fütterer JJ and Barentsz JO: Three-Tesla magnetic resonance-guided prostate biopsy in men with increased prostate-specific antigen and repeated, negative, random, systematic, transrectal ultrasound biopsies: Detection of clinically significant prostate cancers. Eur Urol 62: 902-909, 2012.

13. Hattori S, Kosaka T, Mizuno R, Kanao K, Miyajima A, Yasumizu Y, Yazawa S, Nagata H, Kikuchi E, Mikami S, et al: Prognostic value of preoperative multiparametric magnetic resonance imaging (MRI) for predicting biochemical recurrence after radical prostatectomy. BJU Int 113: 741-747, 2014.

14. Barentsz JO, Richenberg J, Clements R, Choyke P, Verma S, Villeirs G, Rouviere O, Logager V and Fütterer JJ; European Society of Urogenital Radiology: ESUR prostate MR guidelines 2012. Eur Radiol 22: 746-757, 2012.

15. Kikuchi E, Nakashima J, Ishibashi M, Ohigashi T, Asakura H, Tachibana M and Murai M: Prostate specific antigen adjusted for transition zone volume: The most powerful method for detecting prostate carcinoma. Cancer 89: 842-849, 2000.

16. Distler FA, Radtke JP, Bonekamp D, Kesch C, Schlemmer HP, Wieczorek K, Kirchner M, Pahernik S, Hohenfellner M and Hadaschik BA: The value of PSA density in combination with PI-RADS ${ }^{\mathrm{TM}}$ for the accuracy of prostate cancer prediction. J Urol 198: 575-582, 2017.

17. Kubota Y, Kamei S, Nakano M, Ehara H, Deguchi $T$ and Tanaka O: The potential role of prebiopsy magnetic resonance imaging combined with prostate-specific antigen density in the detection of prostate cancer. Int J Urol 15: 322-327, 2008

18. Kosaka T, Mizuno R, Shinojima T, Miyajima A, Kikuchi E, Tanaka N, Shinoda K, Morita S, Mikami S and Oya M: The implications of prostate-specific antigen density to predict clinically significant prostate cancer in men $\leq 50$ years. Am J Clin Exp Urol 2: 332-336, 2014.

19. Daskivich TJ, Fan KH, Koyama T, Albertsen PC, Goodman M, Hamilton AS, Hoffman RM, Stanford JL, Stroup AM, Litwin MS and Penson DF: Effect of age, tumor risk, and comorbidity on competing risks for survival in a U.S. population-based cohort of men with prostate cancer. Ann Intern Med 158: 709-717, 2013.

20. van den Bergh RC, Roemeling S, Roobol MJ, Aus G, Hugosson J, Rannikko AS, Tammela TL, Bangma $\mathrm{CH}$ and Schröder FH: Outcomes of men with screen-detected prostate cancer eligible for active surveillance who were managed expectantly. Eur Urol 55: 1-8, 2009.

21. Tosoian JJ, Trock BJ, Landis P, Feng Z, Epstein JI, Partin AW, Walsh PC and Carter HB: Active surveillance program for prostate cancer: An update of the Johns Hopkins experience. J Clin Oncol 29: 2185-2190, 2011. 